\title{
Synthesis and complexation properties of an amphiphilic cyclodextrin
}

\author{
Carlos J. Gonzalez and Rita H. de Rossi* \\ Instituto de Investigaciones en Físico Química de Córdoba (INFIQC), Departamento de \\ Química Orgánica, Facultad de Ciencias Químicas, Universidad Nacional de Córdoba, Ciudad \\ Universitaria. 5000 Córdoba Argentina \\ E-mail: ritah@dqo.fcq.unc.edu.ar
}

\section{Dedicated to Professor Oswald S. Tee on the occasion of his $60^{\text {th }}$ birthday, and in recognition of his contributions to chemistry in Canada}

(received 01 Sep 01; accepted 08 Nov 01; published on the web 16 Nov 01)

\begin{abstract}
An ester was prepared from the reaction of: 3-dodec-2-enyldihydrofuran-2,5-dione 1 with $\beta$ cyclodextrin giving a compound 2 with an average substitution of 1.2-1.3. This compound self aggregate with a critical aggregation concentration of $1 \times 10^{-4} \mathrm{M}$. It associates with indole derivatives; triptamine, 3 melatonine $\mathbf{4}$ and 3-indolyl acetic acid $\mathbf{5}$ at $\mathrm{pH}$ 7.00. Under these conditions $\mathbf{3}$ is a cation, $\mathbf{4}$ is neutral and $\mathbf{5}$ is an anion, however electrostatic interactions do not seem to be the main driving force for the interaction since the KAss 91,30 and $<10 \mathrm{M}^{-1}$ follow the same order of stability than that reported for native $\beta$-CD. On the other hand, the KAss of 8 anilinonaphtalen-1-sulfonate (ANS) is about the same as that of $\beta$-CD. Using phenolphthalein as indicator, the association equilibrium constant between 2 and $\beta-\mathrm{CD}$ was determined as $2.1 \times 10^{3}$.
\end{abstract}

Keywords: Cyclodextrin, amphiphilic, indole derivatives, 3-dodec-2-enyldihydrofuran-2,5dione

\section{Introduction}

The continuing challenge of using cyclodextrins for solubilization and drug targeting has led to the preparation of a wide variety of chemically modified derivatives in order to improve the properties of this host molecules. ${ }^{1}$ A possible approach as far as pharmaceutical applications are concerned would be to combine the specificity of cyclodextrins with the use of transport properties of organized structures such as vesicles, liposomes and micelles. 
Many amphiphilic cyclodextrins have been described in the literature. Per-6-alkylamino-6deoxy- $\beta$-cyclodextrin derivatives ${ }^{2}$ have been shown to generate highly stable Langmuir-Blodgett films. The formation of amphiphilic cyclodextrins through esterification or etherification at the secondary hydroxyl face has also been described. ${ }^{3}$ However most examples of amphiphilic cyclodextrins are per-6, per-2,3 or per-2,6 substituted compounds and then the inclusion properties of the cavity of cyclodextrin are partially lost due to steric hindrance for the entrance of the host.

Some years $\mathrm{ago}^{4}$ we reported on the preparation of the monoester of $\beta$-cyclodextrin with octadecenyl succinic acid and this compound self associate at very low concentration and binds merocyanine very strongly. Alkenyl succinic esters have many industrial applications ${ }^{5}$ and especially in cosmetics. ${ }^{6}$ Since cyclodextrins have many applications in the cosmetic and pharmaceutical industry, ${ }^{7}$ we thought that it would be interesting to prepare and to study the properties of alkenyl succinic esters of $\beta$-cyclodextrin with lower chain length than those prepared before because they are expected to have better water solubility. Here we report on the preparation and solution properties of an ester of an alkenyl succinic acid with 12 carbon atoms in the chain.

\section{Results and Discussion}

\section{Synthesis of the amphiphilic $\beta$-cyclodextrin.}

The synthesis of the monoester of dodecenyl succinic acid with cyclodextrin is carried out from readily available starting materials. In the first step, eq 1, 1-dodecene is condensed with maleic anhydride through an ene reaction ${ }^{8}$ to give the alkenyl succinic anhydride: 3-dodec-2-enyldihydro-furan-2,5-dione 1.

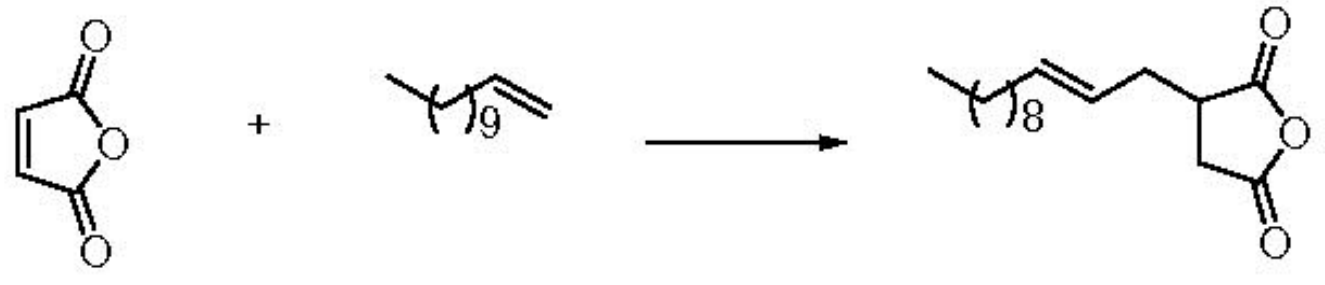

In the second step the ester is formed from the reaction of $\beta$-cyclodextrin with the anhydride to yield the derivatives $\mathbf{2 a}$ and $\mathbf{2 b}$ (CDMod), where $\mathrm{n}$ represents the average degree of substitution, which under the conditions of our reactions is 1.2-1.3. In $\mathbf{2} \mathbf{a}$ and $\mathbf{2} \mathbf{b}$ the numbers 1,2,3 and 12 and the letters a, b, c, and $d$ are to facilitate the description of the NMR spectra of the compounds (see experimental) 


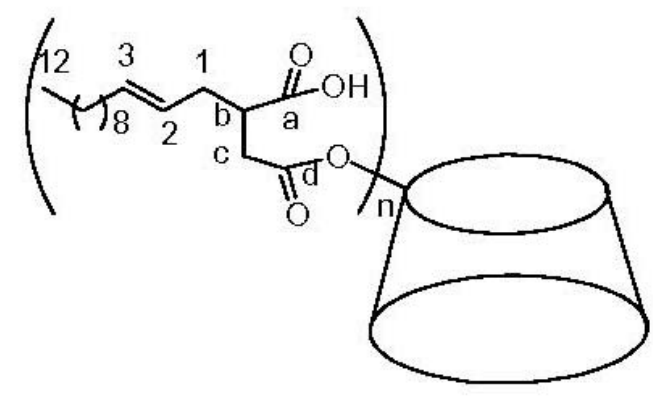

$2 a$

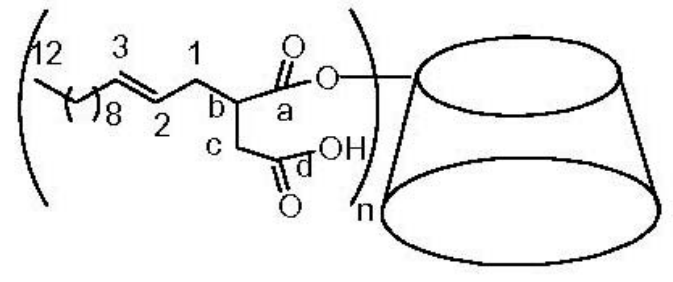

$2 b$

In agreement with the known reactivity of primary and secondary hydroxyl groups ${ }^{9}$ the ester formed predominantly is that of the $\mathrm{OH}$ in position 6 in the glucose units of cyclodextrins and it is at the narrower rim of the cyclodextrin cavity. The fact that is the primary $\mathrm{OH}$ the one that reacts is inferred from the ${ }^{13} \mathrm{C}$ NMR spectrum. The resonance of the $\mathrm{C} 6$ changes from $59.9 \mathrm{ppm}$ in $\beta$-cyclodextrin to 63.61 for CDMod whereas the resonance for $\mathrm{C} 5$ changes from 72.5 to 69.1 ppm. The shift of C6 to lower field and that of C5 to higher fields is known in the literature for compounds substituted in carbon $6 .{ }^{10}$ It was not possible to separate the isomers, so the study reported here was done with the compound with average substitution of 1.2-1.3. In cyclodextrin chemistry the difficulties to separate isomers are well known, so it is common practice to work with mixtures of compounds. ${ }^{11,12}$

\section{Association equilibrium constants}

The addition of CDMod to water solutions containing triptamine (3), melatonine (4) or 3-indolyl acetic acid (5), produces a decrease in fluorescence intensity. All the experiments were done at $\mathrm{pH}=7$ and under these conditions 3 is in the form of the ammonium ion since the pKa is $9.50,{ }^{13} 4$ is neutral and $\mathbf{5}$ is in the form of carboxylate, $\mathrm{pKa}=4.23 .{ }^{14}$ For comparison purposes, the interaction of these substrates was also determined with the alkenyl succinic acid (6) derived from the hydrolysis of $\mathbf{1}$ and also decrease in fluorescence intensity was observed. The decrease in fluorescence intensity in the presence of CDMod contrasts with the results observed with native $\beta$-cyclodextrin $(\beta-C D)$ since in this case the intensity of the fluorescence increases. ${ }^{13,14,15}$ 


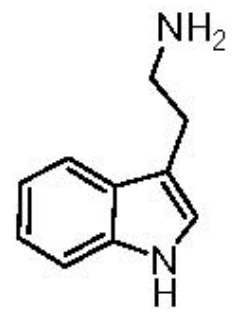

3

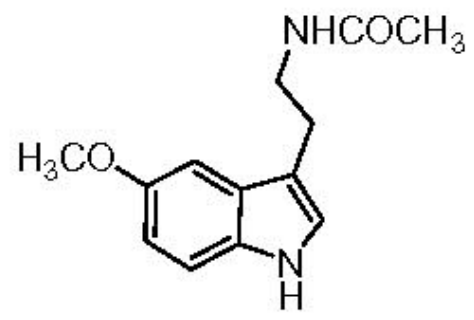

4

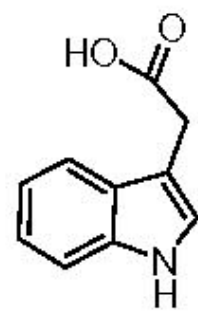

5

In order to explain this observation, $\mathbf{5}$ was added to solutions containing different compounds such as sodium acetate, oleic acid (cis-9-octadecenoic acid), decanoic acid, sodium dodecyl sulfate of variable concentration at $\mathrm{pH}$ 7.00. With the compounds with saturated alkyl chain there was almost no change in fluorescence intensity whereas with oleic acid the fluorescence intensity decreases with concentration of the added acid in similar way as it does with $\mathbf{6}$. For both acids the concentration range used is $1.12 \times 10^{-3}$ to $1.12 \times 10^{-2} \mathrm{M}$. The critical aggregation concentration $(\mathrm{CAgC})$ are $4 \times 10^{-4}$ (see experimental) and $1.5 \times 10^{-3} \mathrm{M}^{16}$ for 6 and oleic acid respectively. The data were plotted using the Stern-Volmer ${ }^{17}$ equation, eq 2

$$
\frac{F_{O}}{F}=1+K_{S V}[Q]
$$

where $F_{o}$ is the fluorescence intensity at a given wavelength of a solution of the substrate at the same $\mathrm{pH}$ but without the quencher, Q, which is $\mathbf{6}$ or oleic acid and $F$ is the value in the presence of Q. From the slope of this plot (not shown) Ksv which is the Stern-Volmer constant is obtained. This value is 54.6 and 31.9 for $\mathbf{6}$ and oleic acid respectively. These results indicate that the quenching of the fluorescence may be attributed mainly to the presence of the insaturation in the chain.

The interaction of CDMod with compounds 3 and $\mathbf{4}$ show a non linear dependence of the intensity of the fluorescence with the concentration of CDMod (Figure 1 is representative) whereas no curvature is observed in the corresponding plot of $\mathbf{5}$.

Figure 1: Effect of the concentration of CDMod on the intensity of fluorescence of triptamine in water at $25^{\circ} \mathrm{C}$ and $\mathrm{pH}=7.00$ (excitation wavelength $279 \mathrm{~nm}$ and emission wavelength $355.5 \mathrm{~nm}$ ) 


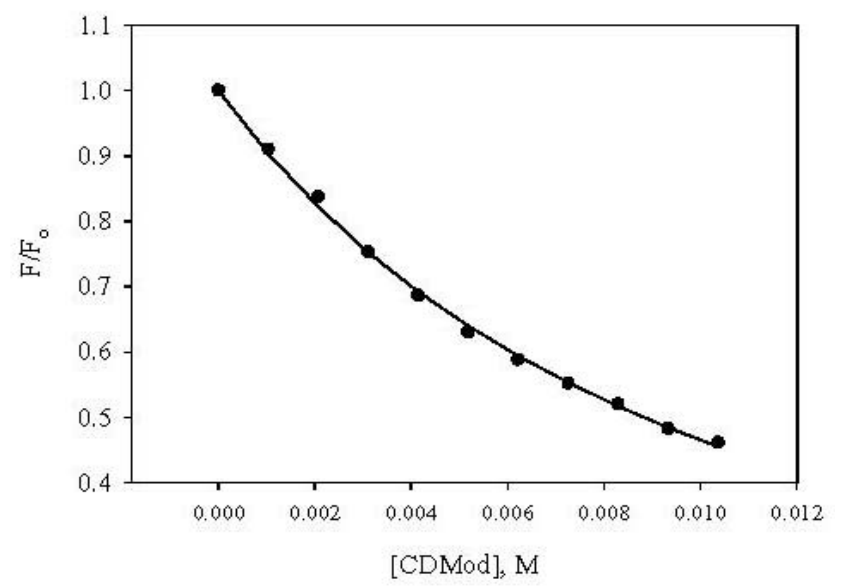

Considering that the free substrate and that associated with the host, either aggregated or not, show independent fluorescence the measured intensity of fluorescence $F$ can be expressed as in eq 3

$$
F=B\left(\phi^{s}{ }_{\varepsilon}^{s}[s]+\phi^{s c} \varepsilon^{s c}[s c]\right)
$$

were $\varphi^{S}$ and $\varphi$ are the fluorescence quantum yield for the substrate (S) and the complex (SC), $\varepsilon^{S}$ and $\varepsilon^{S C}$ represent the extinction coefficient for the substrate and the complex respectively and B is a parameter that depends on the experimental set up.

The 1:1 association equilibrium constant is defined in eq 4 where $\mathrm{C}$ represents the complexing agent.

$$
K_{\text {Ass }}=\frac{[S C]}{[s][C]}
$$

The fluorescence intensity in the absence of $\mathrm{C}, \mathrm{Fo}_{\mathrm{O}}$, is given by eq 5 and the fluorescence intensity $F \sim$ when all the substrate is in the form of the complex is given by eq 6 considering that under all our experimental conditions $[\mathrm{C}] \mathrm{o}>[\mathrm{S}] \mathrm{o} .[\mathrm{C}] \mathrm{o}$ and $[\mathrm{S}] \mathrm{o}$ represent the initial complexing agent and substrate concentration respectively.

$$
\begin{aligned}
& F_{o}=B \phi^{s} \varepsilon^{s}[s]_{O} \\
& F_{\infty}=B \phi^{S C_{\varepsilon}}{ }^{S C}[s]_{O}
\end{aligned}
$$


Combining eq 3-6 and the mass balance for the substrate and for the complexing agent: [S]o $=[\mathrm{S}]+[\mathrm{SC}]$ and $[\mathrm{C}]_{\mathrm{o}}=[\mathrm{C}]$, eq 7 is obtained

$$
\frac{F}{F_{o}}=1+\frac{\left(F_{\infty}-F_{o} / F_{o}\right) K_{\text {Ass }}[C]_{o}}{1+K_{\text {Ass }}[C]_{o}}
$$

Fitting eq 7 to the experimental data allows the determination of the association equilibrium constants for CDMod and $\mathbf{6}$ reported in Table 1. In the case of 3-indolyl acetic acid the KAss can not be determined because there is no curvature in the plot of $F / F O$ vs. Co concentration which indicates that under all our experimental conditions $\mathrm{KAss}[\mathrm{C}] \mathrm{o}<1$. In Table 1 , the values of the association constants for $\beta-\mathrm{CD}$ are also reported for comparison purposes. It is interesting to note that the indole derivatives and phenolphthalein bind less strongly to modified cyclodextrin $\mathbf{2}$ than to native $\beta$ cyclodextrin. Under the $\mathrm{pH}$ of this study, the carboxylic group of CDMod should be ionized and one would expect that $\mathbf{3}$, which is in the form of amonium ion, should bind more strongly to CDMod than to $\beta$-CD. The data in Table 1 shows that the tendency in the binding constant for 3-5 is the same for CDMod and $\beta-C D$ indicating that electrostatic interactions do not make the major contribution to the free energy for the association.

The association of CDMod with phenolphthalein was measured using concentration below the $\mathrm{CAgC}$. The absorption of a solution containing phenolphthalein $6 \times 10^{-6} \mathrm{M}$ at $\mathrm{pH} 10.3$ decreases linearly from 0.157 to 0.139 when CDMod changes from $3.9 \times 10^{-6}$ to $3.9 \times 10^{-5} \mathrm{M}$ with slope -498. Assuming 1:1 association between the host and the guest and considering that as in the association with $\beta-\mathrm{CD},{ }^{18}$ the complex do no absorb at the wavelength of measurement, the association of CDMod with this guest is calculated as $3152 \mathrm{M}^{-1}$. This value is slightly smaller than the value determined using higher concentration of CDMod (see Table 1)

The interaction of 8-anilino-1- naphthalene sulfonate (ANS) 7 with CDMod and with 6 was also studied. It is known that this compound has very weak fluorescence in water but the fluorescence intensity increases considerably in non-polar medium. ${ }^{19}$ In Table 2 , the value of the fluorescence intensity is shown as a function of the additive concentration. 


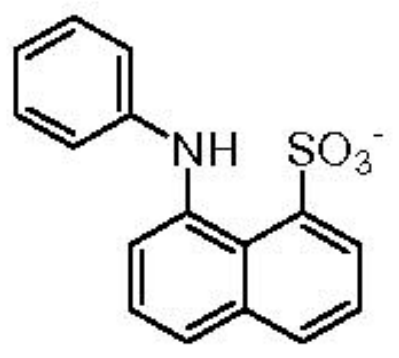

7

It should be noticed that the increase in fluorescence intensity is smaller for $\beta$-CD than for CDMod and 6 indicating that in these two cases the guest is in a microenvironment less polar. On the other hand, there are no significant differences in the association equilibrium constant of the native and modified cyclodextrin (see Table 1). The KAss for $\beta$-CD is in good agreement with literature data, namely $102 \mathrm{M}^{-1}$.

Table 1: Equilibrium association constants $\left(\mathrm{M}^{-1}\right)$ of CDMod, 6 and $\beta$-cyclodextrin with organic guests in water at $25^{\circ} \mathrm{C}$ and at $\mathrm{pH} 7.00$

\begin{tabular}{cccc}
\hline Guest & CDMod & $\mathbf{6}$ & $\beta$-Cyclodextrin \\
\hline Triptamine & $91 \pm 7$ & $155 \pm 24$ & $160 \mathrm{~b}$ \\
Melatonine & $30 \pm 9$ & $\mathrm{~d}$ & $110 \mathrm{e}$ \\
3-Indolyl aceticf & $\mathrm{d}$ & $\mathrm{d}$ & $48 \pm 29 \mathrm{~g}$ \\
ANSh & $89 \pm 7$ & $31 \pm 5$ & $91 \pm 6$ \\
Phenolphthaleini & $(5.1 \pm 0.4) 10_{3 \mathrm{j}}$ & $\mathrm{k}$ & $(1.9 \pm 0.1) 104$ \\
\hline
\end{tabular}

${ }^{\mathrm{a}} \lambda \mathrm{ex}=279 \mathrm{~nm}, \lambda \mathrm{em}=355.5 \mathrm{~nm} .{ }^{\mathrm{b}}$ Data from Reference $13^{\mathrm{c}} \lambda \mathrm{ex}=278 \mathrm{~nm}, \lambda \mathrm{em}=352 \mathrm{~nm} .{ }^{\mathrm{d}}$ Can not be determined. ${ }^{\mathrm{e}}$ Data from Ref. $13{ }^{\mathrm{f}} \lambda \mathrm{ex}=280 \mathrm{~nm}, \lambda \mathrm{em}=365.5 \mathrm{~nm} .{ }^{\mathrm{g}}$ Data from Ref $14{ }^{\mathrm{h}} \lambda \mathrm{ex}$ $=350 \mathrm{~nm}, \lambda \mathrm{em}=519 \mathrm{~nm} .{ }^{\mathrm{i}}$ Determined by measuring the change in absorbance vs. additive concentration at $\mathrm{pH} 10.3$, buffer $\mathrm{HCO} 3 / \mathrm{CO}^{-}{ }^{\mathrm{j}}$ Concentration range $(2.9-29) \times 10^{-5} \mathrm{M} .{ }^{\mathrm{k}}$ No measured 
Table 2: Relative fluorescence intensity of ANS in the presence of CDMod, 6 and $\beta$ cyclodextrin in water at $25{ }^{\circ} \mathrm{C}$ and $\mathrm{pH} 7.00\left(\lambda_{\mathrm{ex}}=350 \mathrm{~nm} \lambda_{\mathrm{em}}=519 \mathrm{~nm}\right)$

\begin{tabular}{cccccc}
\hline CDMod, $10-3 \mathrm{M}$ & $\mathrm{F} / \mathrm{Fo}$ & $\beta-\mathrm{CD}, 10-3 \mathrm{M}$ & $\mathrm{F} / \mathrm{Fo}$ & $\mathbf{6 , 1 0 - 3 \mathrm { M }}$ & $\mathrm{F} / \mathrm{Fo}$ \\
\hline 0 & 1 & 0 & 1 & 0 & 1 \\
1.01 & 32 & 1 & 2.09 & 1.07 & 18.3 \\
2.02 & 55.2 & 2.01 & 2.95 & 2.15 & 35.3 \\
3.03 & 77.4 & 3.01 & 3.86 & 3.22 & 51.4 \\
4.04 & 97.4 & 4.02 & 4.46 & 4.29 & 64.3 \\
5.05 & 114.5 & 5.02 & 5.11 & 5.37 & 80.1 \\
6.06 & 127.7 & 6.02 & 5.77 & 6.44 & 94.1 \\
7.07 & 134.2 & 7.03 & 6.24 & 7.51 & 110.7 \\
8.08 & 153.5 & 8.03 & 6.66 & 8.58 & 123.3 \\
9.09 & 161.3 & 9.04 & 7.07 & 9.66 & 137.6 \\
10.1 & 171.9 & 10 & 7.13 & 10.7 & 147.4 \\
\hline
\end{tabular}

The fact that the association constant of CDMod with all the guests studied here except for ANS, is smaller than the corresponding one for the native $\beta$-CD may be attributed to the self inclusion of the alkenyl chain in the cavity either inter (A) or intramolecularly (B) as shown in the Scheme. This would hinder the entrance of an external guest. It is known that $\beta$-cyclodextrin binds quite strongly to hydrocarbon chains. ${ }^{21}$

The association is evident in the ${ }^{1} \mathrm{H}$ NMR spectrum in $\mathrm{D} 2 \mathrm{O} / \mathrm{NaOD}$. The methyl signal in CDMod is a triplet centered at $1.012 \mathrm{ppm}$ whereas in 6 it is centered at $0.928 \mathrm{ppm}$ on the other hand the signals assigned to the $\mathrm{CH} 2$ groups of carbons 5-11 appear as an unresolved peak at 1.343 in 6 whereas in CDMod it is partially resolved in three peaks at 1.324, 1,379 and 1.478 ppm. This fact may be an indication of the formation of structure $B$ because in this case the different $\mathrm{CH} 2$ are in different microenvironment. Structure $B$ is expected to be more rigid than $A$. A solution of $6\left(2.5 \times 10^{-2} \mathrm{M}\right)$ and $\beta-\mathrm{CD}\left(1.1 \times 10^{-2} \mathrm{M}\right)$ in $\mathrm{D} 2 \mathrm{O} / \mathrm{NaOD}$ shows only one $\mathrm{CH} 2$ signal at $1.330 \mathrm{ppm}$, only slightly shifted up field compared with that of 6 in the absence of $\beta$-CD. On the other hand, the groups of signals in the range of 3.514 to 4.064 which correspond to the hydrogens bonded to carbons 2-6 of the glucose in the $\beta-C D$ ring are sensible modified in CDMod but the peak observed in this case is the same as those observed for the solution of $\mathbf{6}$ and $\beta-C D$. Therefore we think that at least part of CDMod is in solution in the form $B$. 


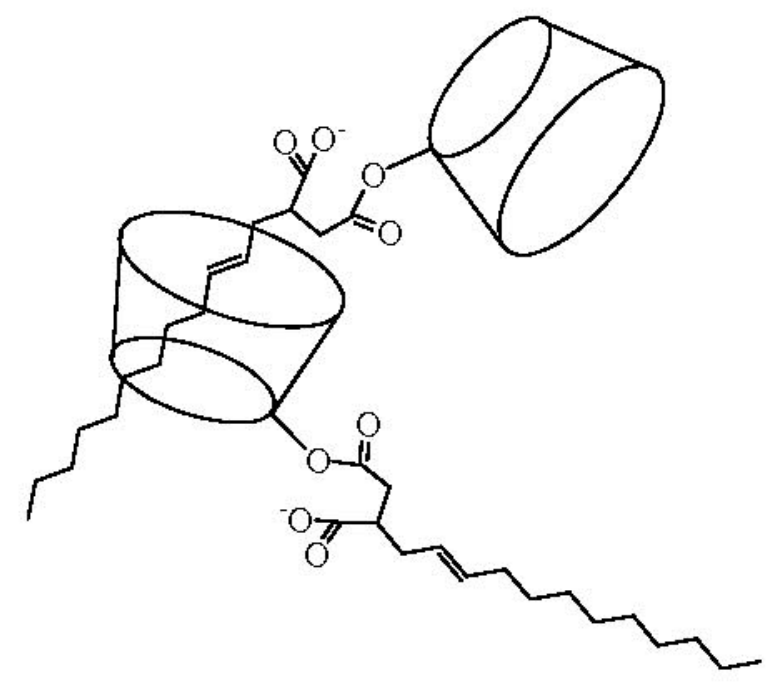

$A$

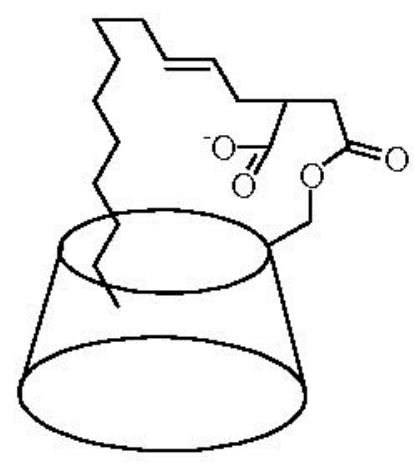

$B$

\section{Scheme}

In order to determine the affinity of the cyclodextrin cavity for the hydrocarbon chain in CDMod, the competitive spectrophotometric technique involving phenolphthalein as indicator usually applied to determine the association of surfactants with $\beta$ $\mathrm{CD}^{22}$ was used.

In solutions containing phenolphthalein $(\mathrm{P}), \beta-\mathrm{CD}$ and $\mathrm{CDMod}$ the equilibria described in eq 8-10 take place. In these equations $\beta$-CD-P and CDMod-P represent the complex of $\mathrm{P}$ with $\beta$-CD and CDMod respectively and $\beta$-CD-CDMod the complex between $\beta$-CD and CDMod. K1, K2 and $\mathrm{K} 3$ are the equilibrium constants defined as in eq

$$
\begin{array}{rll}
P+\beta-C D & \stackrel{K_{1}}{\beta-C D-P} \\
P+\text { CDMod } & \rightleftharpoons \text { CDMod- } \\
\beta-C D+\text { CDMod } & \rightleftharpoons K_{3} & \beta \text {-CD-CDMod }
\end{array}
$$

Since K1 and K2 were previously determined (Table 1), K3 can be determined from the change in absorption of a solution containing constant concentration of $\mathrm{P}$ and CDMod and variable concentration of $\beta-\mathrm{CD}$. The value of $\mathrm{K} 3$ so determined is $2.1 \times 10^{3} \mathrm{M}^{-1}$. This value is ten times smaller than the value of dodecan-1-sulfonate. ${ }^{18}$ The low value of K3 probably indicates that some of the CDMod is in the form $B$ shown in the Scheme. 


\section{Experimental Section}

General Procedures: The FTIR spectra were measured on a Nicolet 5XC spectrophotometer. The NMR spectra were measured in d6-DMSO or in D2O/NaOD on a Brucker AC 200 spectrometer. UV-Vis and spectrofluorimetric determinations were carried out with a Shimadzu UV2101 PC and Jasco FP-777 instruments respectively.

Indole derivatives were purchased in Aldrich or Sigma, phenolphthalein and ANS were samples existing in the laboratory. The purity of all the compounds was checked by determining the melting point and the spectroscopic properties. $\beta$-Cyclodextrin (Roquette, Lestrem, France) was a gift from Ferromet S. A., Buenos Aires, Argentine. Inorganic compounds were analytical grade. Water was purified in a Millipore Milli-Q system. The synthetic procedures were similar to those previously described for octadecenyl succinic anhydride. ${ }^{4}$

3-Dodec-2-enyl-dihydro-furan-2,5-dione 1. Maleic anhydride $51.7 \mathrm{~g}$ ( 0.53 moles) was mixed with $212 \mathrm{~g}$ ( 1.26 moles) of 1 -dodecene and $1.93 \mathrm{~g}$ (17 mmoles) of hydroquinone was added. The reaction was heated in an oil bath at $200{ }^{\circ} \mathrm{C}$ under Nitrogen for 25 hours. After cooling at room temperature, hexane was added to precipitate the unreacted maleic anhydride, after filtering the solid, the hexane was evaporated and the olefin in excess was distilled under vacuum. Yield $123 \mathrm{~g}(88 \%)$. The IR and NMR matched those of the literature. ${ }^{23}$ The product was used without further purification for the esterification of $\beta$-cyclodextrin.

2-Dodec-2-enyl-succinic acid 6. To $20.1 \mathrm{~g}(0.0756 \mathrm{~mol})$ of the anhydride $120 \mathrm{ml}$ of a solution of $\mathrm{NaOH} 6 \mathrm{M}$ was added and the mixture was heated at $90-100{ }^{\circ} \mathrm{C}$ for one hour. After cooling, $\mathrm{HCl}$ was added to acidify the solution and then extracted with ethyl ether. The ether extract was washed several times with water and then evaporated. The dried residue gave NMR and IR spectrum in agreement with literature data.

\section{Esterification of $\beta$-cyclodextrin}

The $\beta$-cyclodextrin is previously purified in a Soxhlet with benzene during 24 hours and then dried in vacuum at $50{ }^{\circ} \mathrm{C}$. The anhydride $1,1.04 \mathrm{~g}(3.93 \mathrm{mmol})$ dissolved in $25 \mathrm{~mL}$ of anhydrous pyridine is added at once to the dried cyclodextrin, $2.1 \mathrm{~g}$ (1.85 mmole) dissolved in $25 \mathrm{~mL}$ of pyridine at $95-100{ }^{\circ} \mathrm{C}$ and then additional $25 \mathrm{~mL}$ of the solvent are added. The solution is heated during 8 hours and then $10 \mathrm{~mL}$ of water is added. The solvent is evaporated and the crude product extracted in a Soxhlet with acetone during 24 hours. The dried residue is washed with water at $\mathrm{pH} 2$, then with water until the $\mathrm{pH}$ reach 5-6 and finally placed in a Soxhlet with acetone during approximately 16 hours. Yield $1.934 \mathrm{~g}$. FAB (Trietilendiamine matrix) $1423 .{ }^{13} \mathrm{C}$ NMR (d6-DMSO) $\delta(\mathrm{ppm}$ ) 13.98 (CH3), 22.09-34.58 (five poorly resolved peaks -CH2), 60.03 (C6 of the unsubstituted glucoses of CDMod), 63.61 (C6 of the substituted glucose) 69.1 (C5 of the substituted glucose), 72.11 (C2 of cyclodextrins), 72.5 (C5 of non substitued glucose) 73.14, 
81.65 and 102.03 (C3, C4 and $\mathrm{C} 1$ of cyclodextins), 123.97 (C2 in structure 2b), 126.21 (C2 in structure 2a), 133.24 (C3 in structure 2a) 136.24 (C3 in structure 2b) 171.67 (Cd in 2a), 172.97 (Ca in 2b), $173.6(\mathrm{Cd}$ in $\mathbf{2 b}) ; 175.15$ (Ca in 2a).

${ }^{1} \mathrm{H}$ NMR (d6-DMSO) $\delta$ (ppm) 0.884 (unresolved triplet, -CH3), 1.256 (CH2 5 to 11), 1.9-2.5) (poorly resolved peaks attributed to $\mathrm{CH} 21,4, \mathrm{~b}$, and c), 3.37-3.66 (CH of carbons 2,3,4,5, and 6 of the cyclodextrin ring), 4.42 (primary $\mathrm{OH}$ of cyclodextrin), 4.86 (C1-H of cyclodextrin), 5.36 (poorly resolved multiplet assigned to the vinilic hydrogens 2 and 3), 5.65 hydrogen of the secondary $\mathrm{OH}$ of cyclodextrin.

IR in $\mathrm{KBr}\left(\mathrm{cm}^{-1}\right) 3336$ (OH of cyclodextrin), $1741(\mathrm{C}=\mathrm{O}$ ester $)$.

Attempts to purify the product using Shephadex G15 were unsuccessful therefore we determined the average substitution integrating the $\mathrm{CH}$ groups of the chain compared with $\mathrm{C}-1$ of the cyclodextrin. The average substitution ratio was always 1.2-1.3 and this product was used for the equilibrium determinations. No significant differences in equilibrium association constants were observed within products coming from independent synthesis.

Equilibrium determinations. All the determinations were done at $25{ }^{\circ} \mathrm{C}$ using water as solvent. Spectrofluorimetric method. Two solutions were prepared and mixed in different proportions such as to have in the desired concentration of the substrate and host. One of the solution contain the substrate, typically $1.0 \times 10^{-5} \mathrm{M}$ in buffer (H2PO4 /HPO4) at $\mathrm{pH}=7.00$ and the other contain the substrate at the same concentration and $\mathrm{pH}$ and the host at the maximum concentration. The intensity of fluorescence at one wavelength (F) was measured using the appropriate excitation wavelength as indicated in Table 1. The equilibrium constants were calculated by fitting eq 7 to the data. When 6 was the host the concentration used was the analytical concentration minus the $\mathrm{CAgC}$. The concentration range used was $(1-10) \times 10^{-3} \mathrm{M}$

Spectrophotometric method. This was used for the determinations using phenolphthalein as guest. Solutions absorptions were obtained at $552 \mathrm{~nm}$ in phenolphthalein solutions $6.5 \times 10^{-6} \mathrm{M}$ buffered at $\mathrm{pH} 10.2$ with $0.05 \mathrm{M} \mathrm{NaHCO} 3 / 0.10 \mathrm{NaOH}$ solution. A precise $3 \mathrm{~mL}$ volume of this solution was placed in the cuvette and titrated with a solution containing $1.3 \times 10^{-3} \mathrm{M} \beta-\mathrm{CD}$ or $8.8 \times 10^{-3} \mathrm{M}$ CDMod and phenolphthalein $6.5 \times 10^{-3} \mathrm{M}$ in 10 additions (10 $\mu \mathrm{L}$ each) until the pink color notably diminished. The equilibrium constant K1 (eq 8) was determined by fitting the experimental data to eq $11^{24}$

$$
A_{o}-A=\frac{\varepsilon_{p} K_{1}[P]_{o}[\beta-C D]_{O}}{1+K_{1}[\beta-C D]_{O}}
$$


For the determination of K3 (eq 10) the solution in the cuvette contained also CDMod $5 \times 10^{-4} \mathrm{M}$ and it was titrated with $\beta-\mathrm{CD} 1.5 \times 10^{-2} \mathrm{M}$. The calculation of K3 was done considering eq 8-10 and the mass balance for the system.

Critical aggregation concentration. This value was determined for CDMod and for 6. The intensity of fluorescence of a solution of melatonine $1.0 \times 10^{-5} \mathrm{M}$ at $\mathrm{pH}=7.00$ was measured as a function of the concentration of the added surfactant $\left(1 \times 10^{-7}-1 \times 10^{-3} \mathrm{M}\right)$. The measured $\mathrm{F}$ value was aproximatelly constant up to a point where there was a significant change in intensity. The CAgC for CDMod was $1.23 \times 10^{-4} \mathrm{M}$ and for $64.13 \times 10^{-4} \mathrm{M}$.

\section{Acknowledgements}

Financial support by Consejo Nacional de Ciencia y Tecnica (CONICET), Argentina, the Agencia Cordoba Ciencia, and the SECYT, Universidad Nacional de Cordoba is gratefully acknowledged.

\section{References and Notes}

1. See for example: (a) Saenger, W. Angew. Chem. Int. Ed. 1980, 19, 344. (b) Khan, A. R.; Keith, J. S.; D'Souza, V. T. Chem. Rev. 1998, 98, 5.

2. (a) Yabe, A.; Kawabata, Y.; Niino, H.; Tanaka, M.; Ouchi, A.; Takahashi, H.; Tamura, S.;

Tagaki, W.; Nakahara, H.; Fukuda, K. Chem.Lett. 1988, 1. (b)Yabe, A.; Kawabata, Y.; Niino, H.; Matsumoto, M.; Ouchi, A.; Takahashi, H.; Tamura, S.; Tagaki, W.; Nakahara, H.; Fukuda, K. Thin Solid Films 1988, 160, 33.

3. (a) Parrot-Lopez, H.; Ling, C.; Zhang, P.; Baskin, A.; Albrecht, G.; De Rango, C.; Coleman, A. W. J Amer Chem Soc 1992, 114, 5479. (b) Coleman, A. W.; Kasselouri Supramol Chem 1993, 1, 155. (c) Tchoreloff, P. C.; Boissonnade, M. M.; Coleman, A. W.; Baszkin, A. Langmuir 1995, 11, 191.

4. Gonzalez, C. J.; de Rossi, R. H.; Seldes, A. Anal. Asoc. Quim. Argentina 1998, 86, 299.

5. (a) Evans, E. M.; Hughes, A. Brit. Patent 1,176,094, 1970. (b)Roger, T. Fr. Patent 1,516,856, 1968.

6. Sato, N.; Yahagi, K.; Tsujii, K. Jpn. Patent 86-166083, Chem. Abstr. 110: 121039t, 1988.

7. See for instance Cyclodextrins News, J. Szejtli, Ed; Cyclolab: Budapest, Hungary, 2001, 15.

8. Opolzer, W. Angew. Chem. Int. Ed. 1984, 23, 876.

9. Khan, A. R.; Forgo, P.; Stine, K. J.; D'Souza, V. T. Chem Rev 1998, 98, 1977.

10. (a) Shen, Z.; Huang, S. L.; Tsai, G.; Chen, Z.; Tsao, G.T. Carbohydrate Research 1990, 201, 
241. (b) Tong L.-H.; Hou Z.; Inoue Y.; Tai A. J. Chem. Soc., Perkin Trans 2 1992, 1253.

11. Natoli, M.; Pagliero, C.; Trotta, F.; Drioli, E. J. Mol. Catal. A Chem. 1997, 121, 179.

12. Commercially available substituted cyclodextrin such as the commonly used hydroxipropyl$\beta$-cyclodextrin are also mixtures of isomers.

13. Galian, R. E., Veglia, A. V., de Rossi, R. H. Analyst 1998, 123, 1587.

14. Sanrame, C. N.; de Rossi, R. H.; Arguello, G. A. J. Phys. Chem. 1996, 100, 8151.

15. Galian, R. E., Veglia, A. V., de Rossi, R. H, Analyst 2000, 125, 1465.

16. Drew Myers, Surfactant Science and Technology, $2^{\text {nd }}$ Edn; VCH, 1993; p 112.

17. Lakowics, J. Principles of Fluorescence Spectroscopy, Plenun Press: NY, 1983; p 25.

18. Gray, J.E.; Maclean, S.A.; Reinsborough, V.C. Aust. J. Chem. 1995, 48, 551.

19. Schneider, H. J.; Blatter T.; Simova S. J. Amer. Chem. Soc. 1991, 113, 1996.

20. Liu, Y.; You, C-C J. Phys Org. Chem. 2001, 14, 11.

21. Rekharsky, M. V.; Inoue, Y. Chem. Rev. 1998, 98, 1875.

22. SAsaki, K. J., Christian, S. D. Tucker, E. E. J. Colloid Interface Sci. 1990, 134, 412.

23. (a) The Aldrich Library of FTIR spectra, Edition 1, C. J. Puchert, Vol 1, 717 A. (b) The Aldrich Library of ${ }^{13} \mathrm{C}$ and ${ }^{1} \mathrm{H}$ NMR spectra C. J. Pouchert and J. Behnke, Vol. 1, 1177A.

24. Connors, K. A Binding Constants: The Measurement of Molecular Complex Stability. John. Wiley \& Sons: New York, 1987; p 148. 